Supporting information for:

\title{
Catalytic Imido-Transfer Reactions of Well-Defined Silica-Supported Titanium Imido Complexes Prepared via Surface Organometallic Chemistry
}

Pavel A. Zhizhko, ${ }^{,+\dagger}$ Andrey V. Pichugov, ${ }^{\dagger, \neq}$ Nikolai S. Bushkov, ${ }^{+, \uparrow}$ Andrey V. Rumyantsev, ${ }^{\dagger, \boldsymbol{\tau}}$ Kamil I. Utegenov, ${ }^{\dagger}$ Valeria N. Talanova, ${ }^{\dagger}$ Tatyana V. Strelkova, ${ }^{\dagger}$ Dmitry Lebedev, ${ }^{\S}$ Deni Mance, ${ }^{\S}$ Dmitry N. Zarubin, ${ }^{,+}$

${ }^{\dagger}$ A. N. Nesmeyanov Institute of Organoelement Compounds, Russian Academy of Sciences, Vavilov str., 28, 119991 Moscow, Russia

${ }^{\ddagger}$ Higher Chemical College, D. Mendeleev University of Chemical Technology of Russia, Miusskaya sq., 9, 125047 Moscow, Russia

'Chemistry Department, Moscow State University, Vorob'evy Gory, 1, 119991 Moscow, Russia

${ }^{\S}$ Department of Chemistry and Applied Biosciences, ETH Zürich, Vladimir-Prelog Weg, 1-5, $\mathrm{CH}-8093$ Zürich, Switzerland 


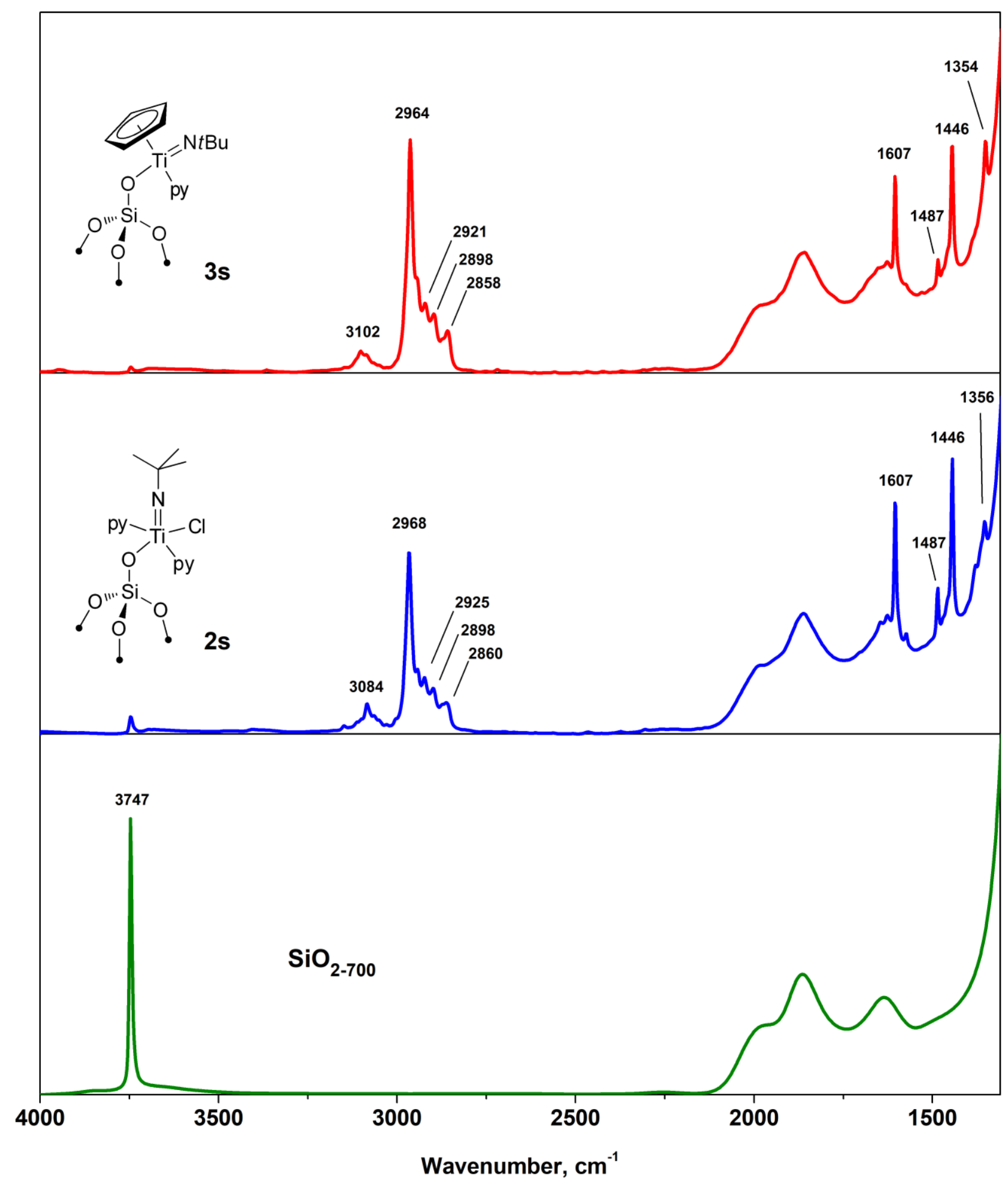

Fig. S1. IR spectra of $\mathrm{SiO}_{2-700}$ and grafted materials $\mathbf{2 s ,} 3 \mathbf{s}$. 


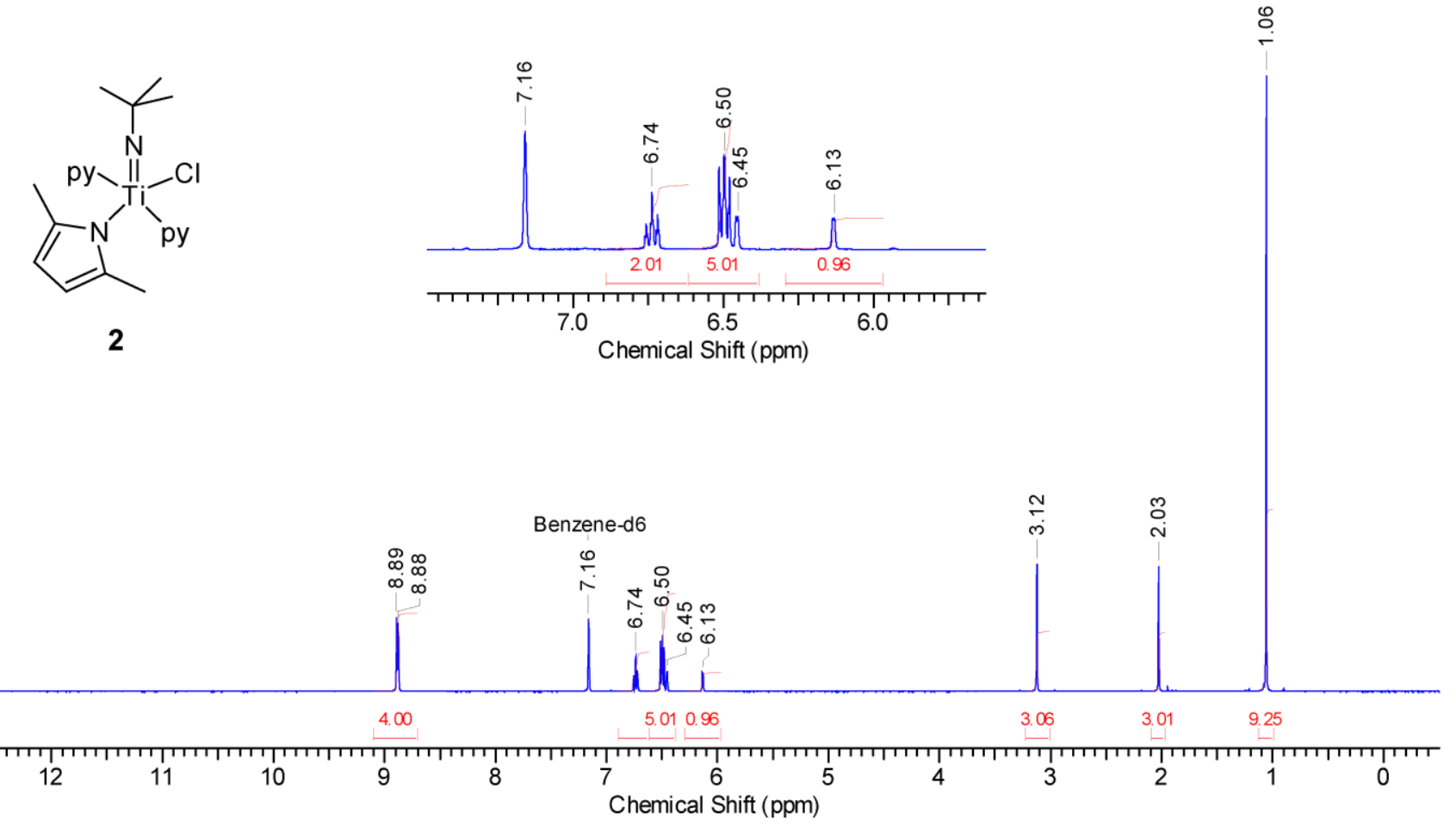

Fig. S2. ${ }^{1} \mathrm{H} N M R$ spectrum of $\left[\mathrm{Ti}(=\mathrm{N} t \mathrm{Bu}) \mathrm{Cl}\left(\mathrm{Me}_{2} \mathrm{Pyr}\right)(\mathrm{py})_{2}\right](2)$ in $\mathrm{C}_{6} \mathrm{D}_{6}$.

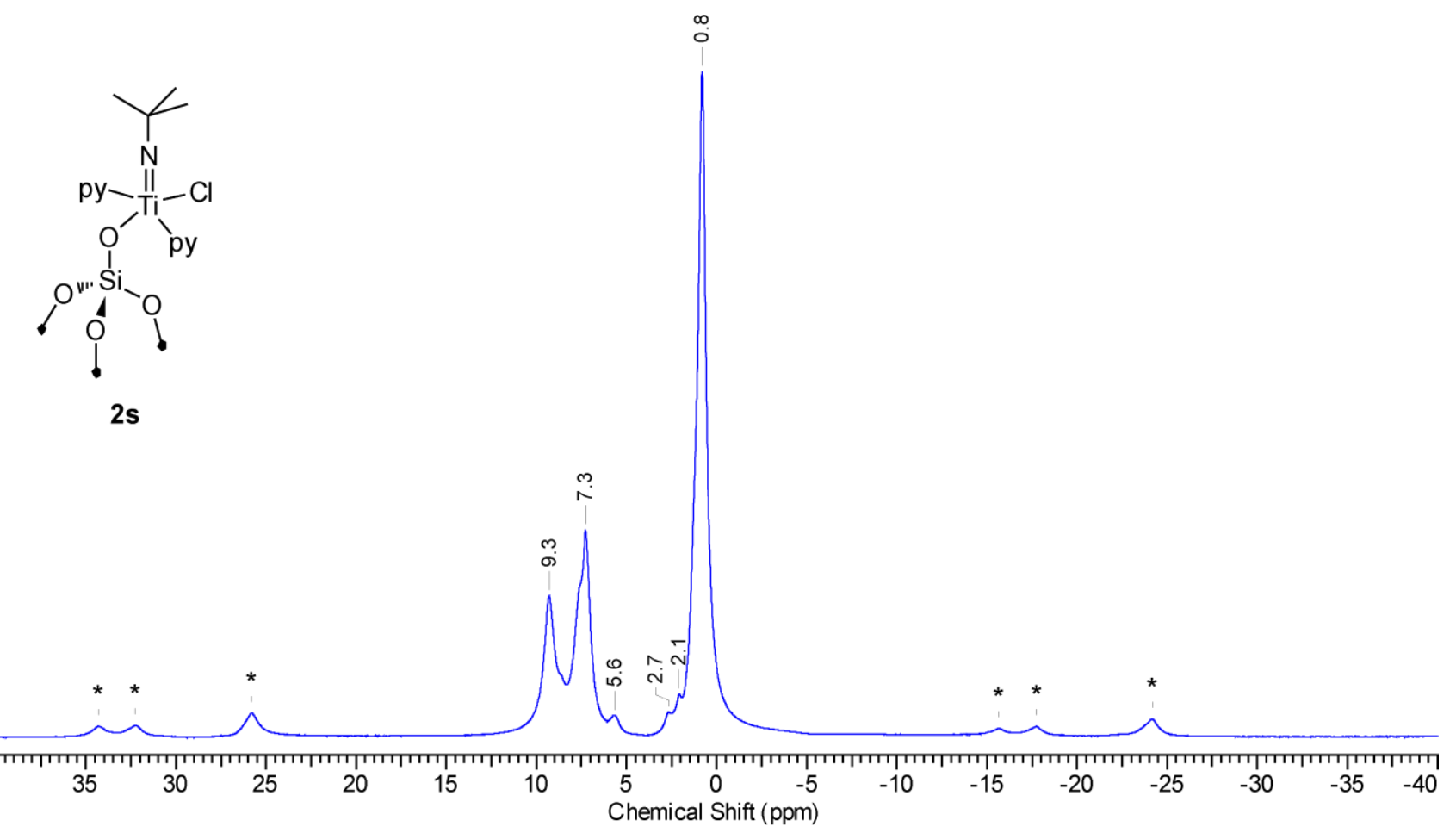

Fig. S3. ${ }^{1} \mathrm{H}$ MAS solid-state $\mathrm{NMR}$ spectrum of $\left[(\equiv \mathrm{SiO}) \mathrm{Ti}(=\mathrm{NtBu}) \mathrm{Cl}(\mathrm{py})_{2}\right]$ (2s) (400 MHz; MAS frequency $10 \mathrm{kHz}$ ). Asterisks indicate spinning side-bands. 


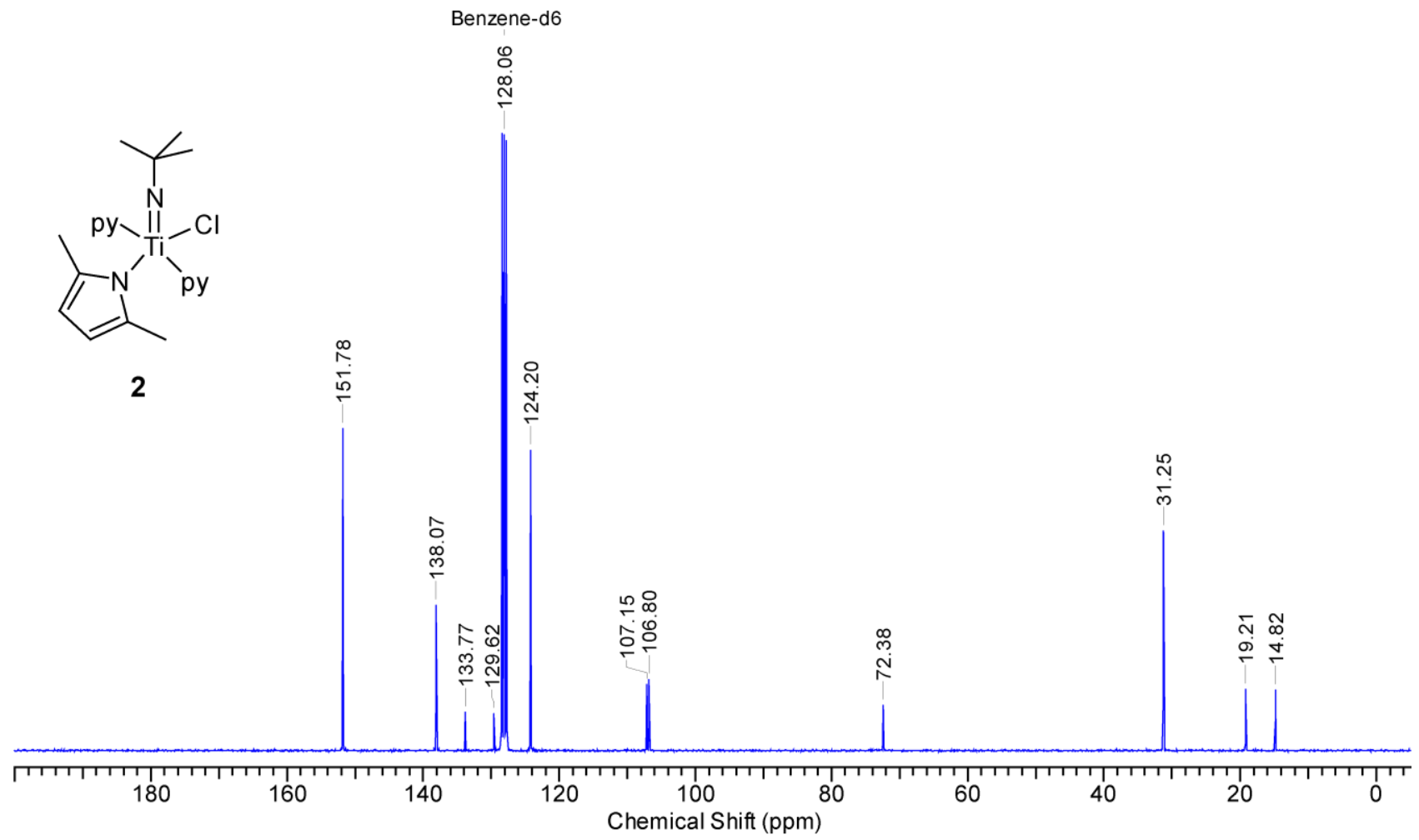

Fig. S4. ${ }^{13} \mathrm{C}$ NMR spectrum of $\left[\mathrm{Ti}(=\mathrm{NtBu}) \mathrm{Cl}\left(\mathrm{Me}_{2} \mathrm{Pyr}\right)(\mathrm{py})_{2}\right](2)$ in $\mathrm{C}_{6} \mathrm{D}_{6}$.

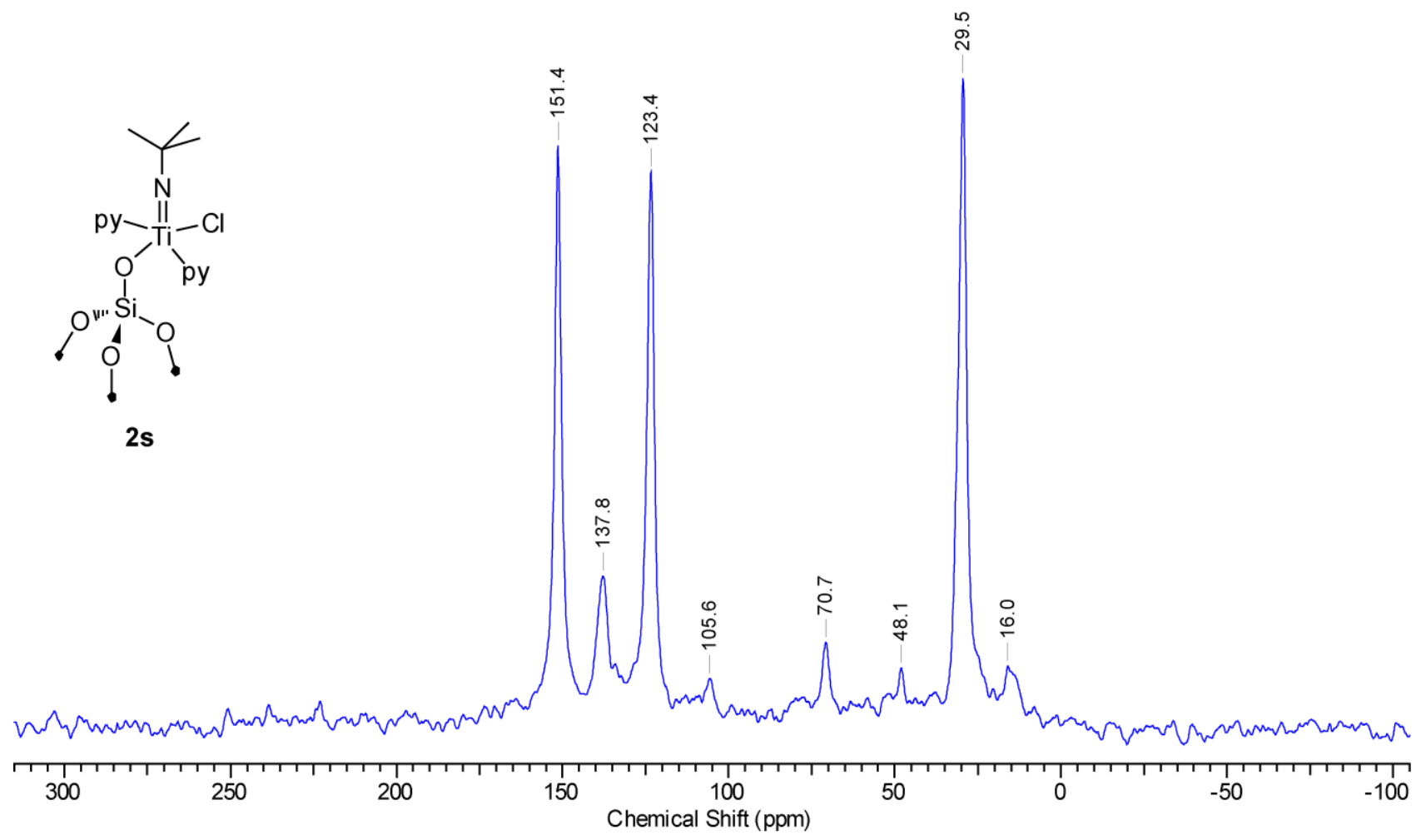

Fig. S5. ${ }^{13} \mathrm{C}$ CP MAS solid-state NMR spectrum of $\left[(\equiv \mathrm{SiO}) \mathrm{Ti}(=\mathrm{NtBu}) \mathrm{Cl}(\mathrm{py})_{2}\right]$ (2s) $(100 \mathrm{MHz}$; MAS frequency $10 \mathrm{kHz}$; CP contact time $3 \mathrm{~ms}$; recycle delay 2 s; 33'000 scans; LB 120). 


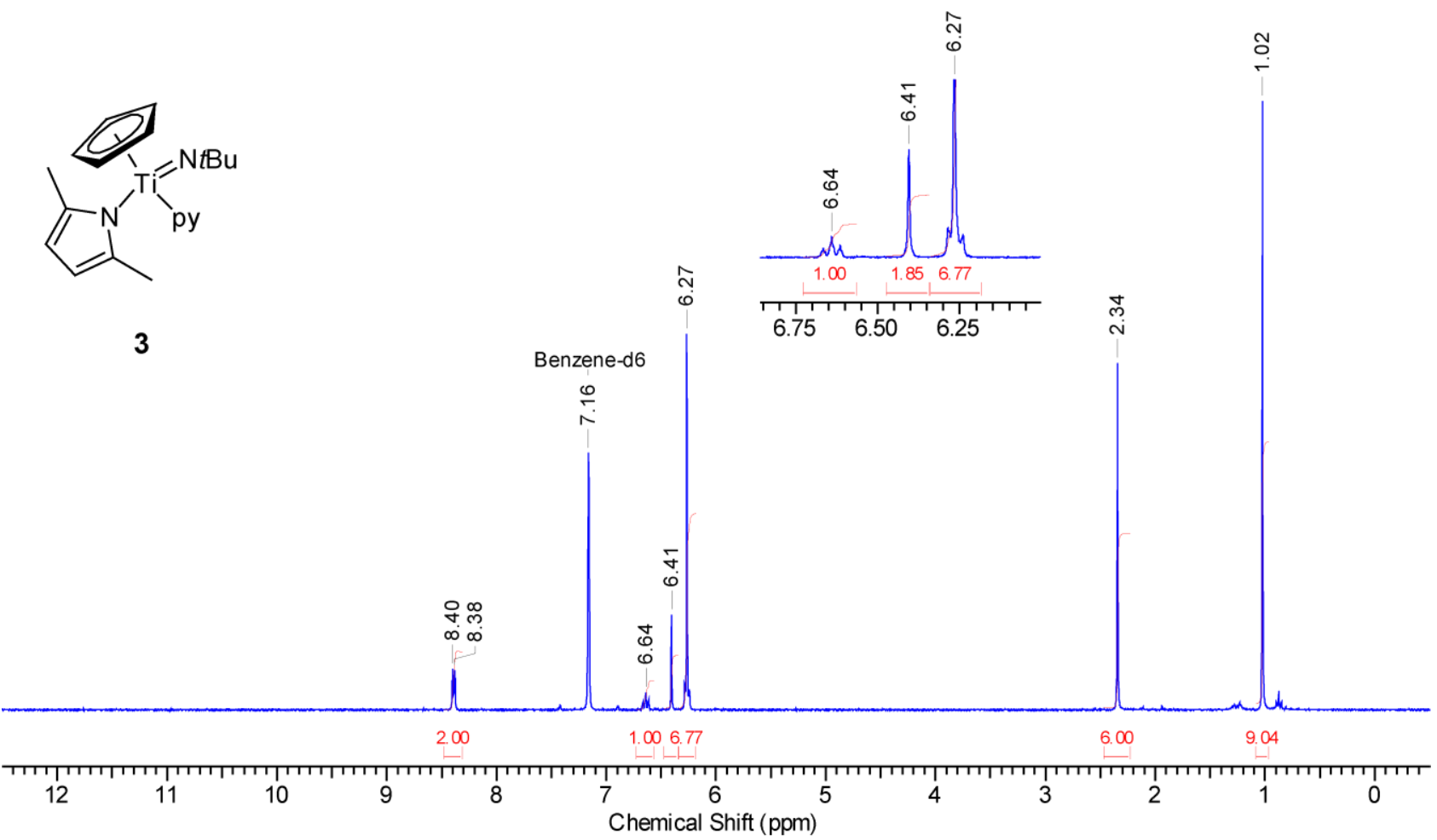

Fig. S6. ${ }^{1} \mathrm{H}$ NMR spectrum of $\left[\mathrm{Ti}(=\mathrm{NtBu}) \mathrm{Cp}\left(\mathrm{Me}_{2} \mathrm{Pyr}\right)(\mathrm{py})\right](3)$ in $\mathrm{C}_{6} \mathrm{D}_{6}$.

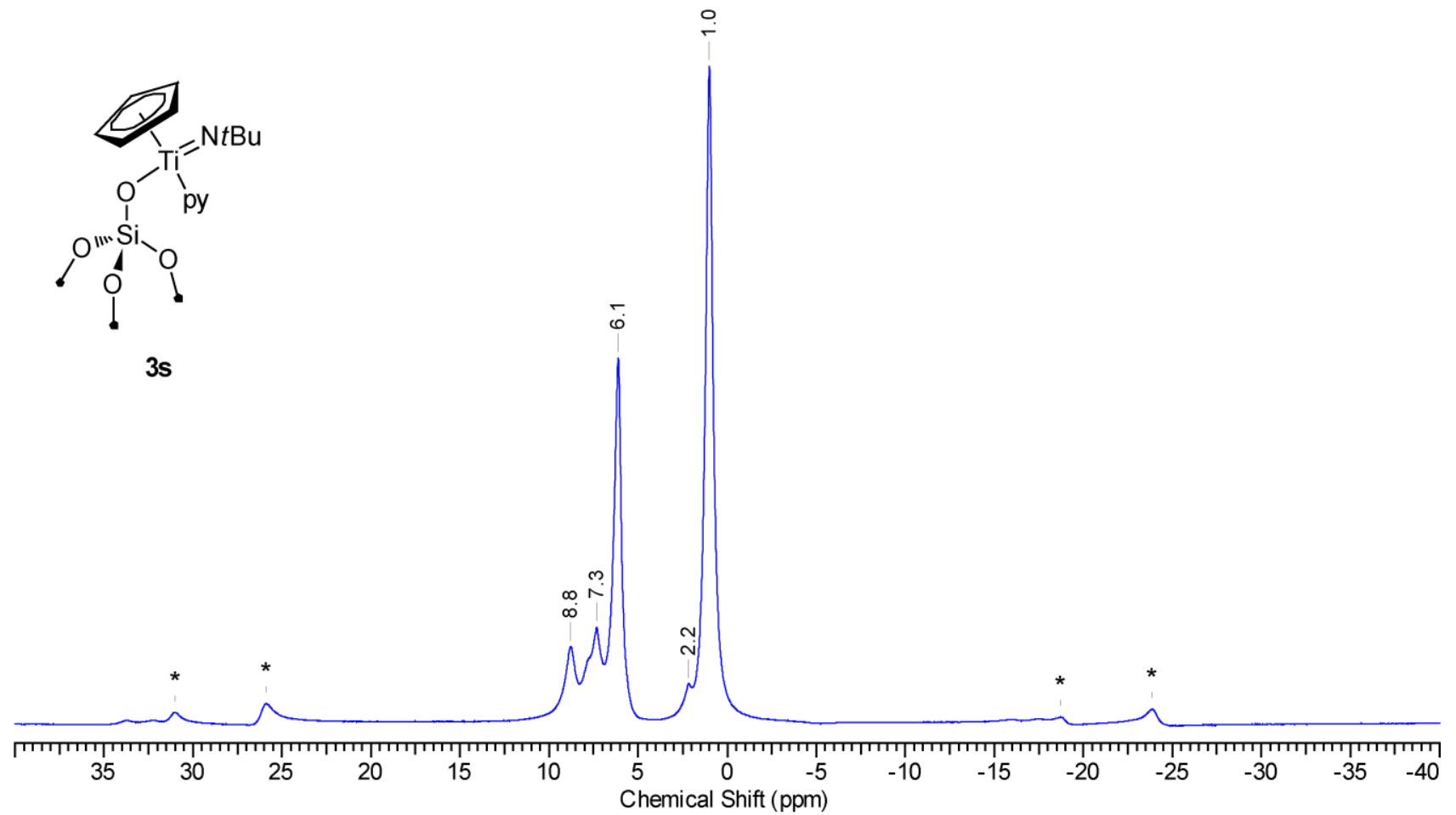

Fig. S7. ${ }^{1} \mathrm{H}$ MAS solid-state NMR spectrum of $[(\equiv \mathrm{SiO}) \mathrm{Ti}(=\mathrm{NtBu}) \mathrm{Cp}(\mathrm{py})]$ (3s) $(400 \mathrm{MHz}$; MAS frequency $10 \mathrm{kHz}$ ). Asterisks indicate spinning side-bands. 


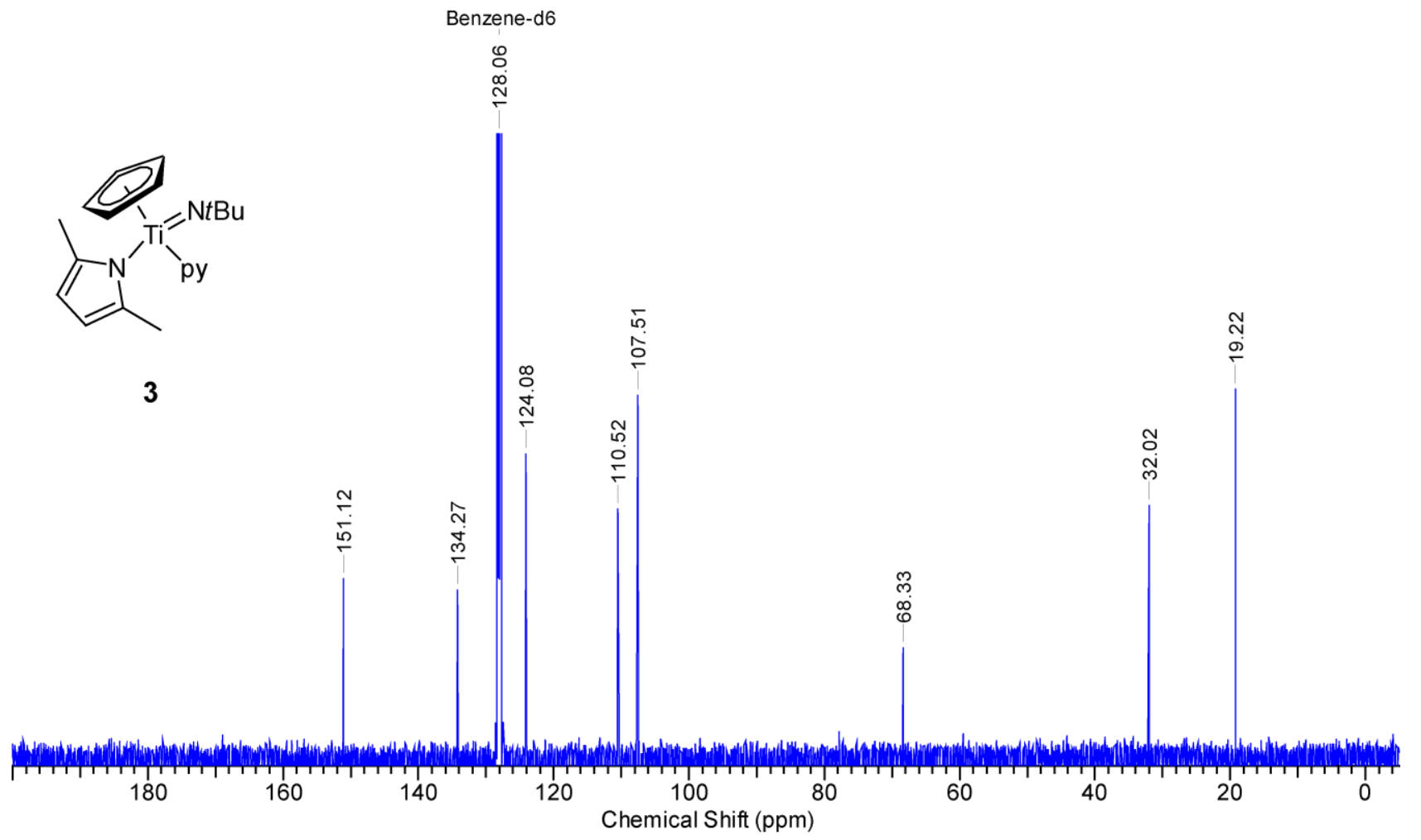

Fig. S8. ${ }^{13} \mathrm{C} N M R$ spectrum of $\left[\mathrm{Ti}(=\mathrm{NtBu}) \mathrm{Cp}\left(\mathrm{Me}_{2} \mathrm{Pyr}\right)(\mathrm{py})\right](\mathbf{3})$ in $\mathrm{C}_{6} \mathrm{D}_{6}$.

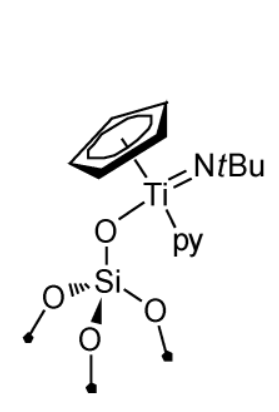

3s

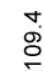
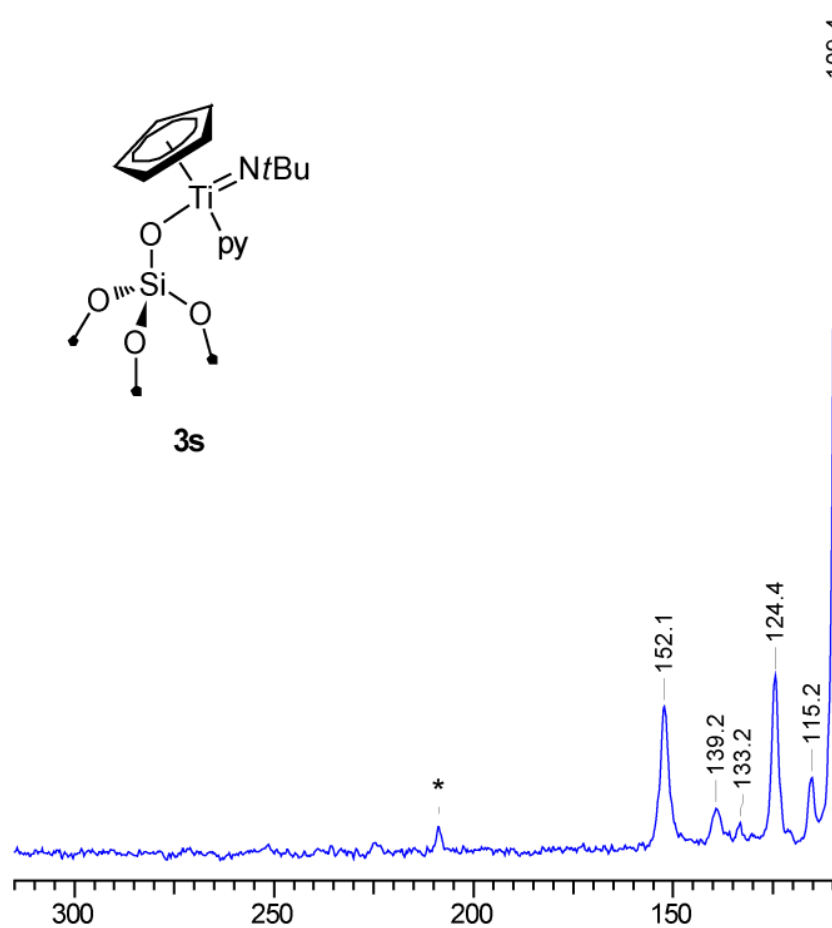